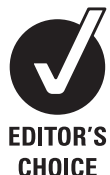

CHOICE

\title{
Pitfalls in the use of multicolour flow cytometry in haematology
}

\author{
Ulrika Johansson, ${ }^{1}$ Marion Macey ${ }^{2}$
}

${ }^{1}$ Haematology Department, University Hospital's Bristol NHS Foundation Trust, Bristol, UK ${ }^{2}$ Department of Haematology, Bart's and The Royal London NHS Trust, London, UK

\section{Correspondence to}

Marion Macey, Department of Haematology, Bart's and

The London NHS Trust, London, UK; marion.macey@ bartsandthelondon.nhs.uk

Accepted 6 March 2011 Published Online First 30 March 2011

\begin{abstract}
Multicolour flow cytometry in haematology has developed considerably in recent years. The ability to analyse eight or more colours of fluorescence on millions of cells in a matter of minutes has enabled the provision of rapid and reliable measures of minimal residual disease for clinicians. The use of multicolour analysis has also enabled more specific characterisation of presenting leukaemias and lymphomas. However, there has not been a concomitant increase in the knowledge and experience of the flow cytometrists to deal with certain problems associated with this more complex analysis.
\end{abstract}

\section{INTRODUCTION}

Flow cytometry was developed in the early to mid 1900s and first used in a haematological clinical setting in the 1980s. Over the past 30 years the instruments have become smaller but are much more sophisticated. However, this development has not been accompanied by the knowledge required for the standardised set-up and use of these complex instruments. Recently, a number of groups have begun to develop guidelines. ${ }^{1-4}$ These guidelines stress the importance of training and education, and some notable societies including the Royal Microscopical Society, the Institute of Biomedical Sciences and, most recently, the International Society for Advancement of Cytometry, have all developed certificated training courses. To date a number of pitfalls in the interpretation of multicolour flow cytometry in haematology have become evident and these are discussed below.

\section{ANTISERA AND FLUOROCHROME COMBINATIONS: ARTIFACTUAL RESULTS}

The move from single or dual immunofluorescence analysis to the use of $8+$ fluorochrome labelled antibodies has led to an increase in the amount of information regarding the subpopulations of cells within a particular sample, and has proven to be of great benefit for the detection of minimal residual disease in patients with relapsed leukaemia; however, determining the correct combination of fluorochromes to use can be problematic. ${ }^{5}$ In cases where the expression level of an antigen is of clinical significance, it is important to use a fluorochrome that has a high spectral emission but does not compromise the read-out of other fluorochrome detectors due to its potentially high spectral overlap. Steric hindrance may also be a problem when two antibodies are used to label antigens that are in very close proximity to each other on the cell surface. This leads to reduced binding of the 'hindered' antibody and a reduction in the associ- ated fluorescence, resulting in a lower than expected expression. Fluorescence resonance excitation transfer may also occur between two adjacently placed fluorochromes and has been observed when labelling cells with, for example, both phycoerythrin (PE)-conjugated antibodies and tandem dye-conjugated antibodies such as allophycocyanin (APC)-Cy7, PE-Cy5 or PE-Cy7. In this situation the fluorescence from the PE-conjugated antibody is quenched and that from the tandem antibody is enhanced, again giving erroneous apparent levels of expression for the antigens investigated. Moreover, if the PE-conjugated antibody is adjacent to the APC-Cy7 conjugated antibody, fluorescence resonance excitation transfer would result in a false PE-Cy7 signal. To overcome the problems associated with using multiple combinations of fluorochromes it is advisable to perform a set of experiments termed 'fluorescence minus one' or FMO. ${ }^{5-7}$ Here, one fluorochromeconjugated antibody is left out at a time. This test is also helpful for determining the background fluorescence level in each channel. Another useful test is to compare the fluorescence intensity and expression patterns in the multicolour assay with those of each antibody alone. This helps to determine whether any changes in fluorescence occur when the antibodies are combined.

\section{TANDEM FLUOROCHROMES}

Tandem dyes were developed to increase the number of fluorochromes that could be excited by a given wavelength associated with the lasers/ diodes commonly found in flow cytometers - that is, argon blue $488 \mathrm{~nm}$, helium neon red $633 \mathrm{~nm}$ and helium cadmium violet $405 \mathrm{~nm}$. Tandem dyes are conjugates of two fluorochromes and they frequently require larger compensation settings for spectral overlap than other dyes. ${ }^{8}$ Compensation is used to correct for the spectral overlap that occurs when the fluorescence from one fluorochrome enters the detector for another fluorochrome. It is a commonly held view that inaccurate compensation is the main source of erroneous data in multicolour flow cytometry. Clinical laboratories often use generic fluorochrome-conjugated antibodies for setting compensation; for example, CD8fluorescein isothiocyanate (FITC) may be used for any FITC-conjugated antibody. For tandem-conjugated antibodies however, the amount of correction needed regularly varies from batch to batch, so considerably more care is needed when setting the compensation for multicolour work. Also, it is important that they are not exposed to light or conditions that would break the coupling between the two dyes, which would result in reduced and/or 
erroneous fluorescence signals. ${ }^{79}$ Even so, there are some data that show APC-tandem degradation to be cell-dependent, and the effect is reduced by inhibiting the metabolic activity of the cells by, for example, adding sodium azide to the buffer. ${ }^{10}$

\section{LABELLING PROCESS}

Any assay carries with it a certain risk of human error and, in the case of $8+$ colour cytometry, pipetting the wrong antisera or omitting one of 10 antibodies is such a possibility. If an antibody has been omitted entirely (by accident), this can often be identified by the absence of fluorescence associated with a cell population in a normal peripheral blood sample, for example CD8+ lymphocytes. More difficult may be a lack of fluorescence associated with myeloid progenitors in a post-transplant marrow, where few precursors are available and data may be interpreted as no progenitors being present. Knowledge of both normal non-specific and specific binding patterns is crucial to identify these errors. To avoid this issue, a cocktail containing sufficient antibodies for several tests can be produced and used over a short period of time. There is some debate regarding the stability of certain fluorochromes in such a cocktail, in particular the APC-tandem dyes. As yet there are no published data on this, but the authors have preliminary data suggesting that cocktails containing three tandem dyes are stable for at least one month. The risk for human error is also increased by the frequent stocking of any given monoclonal antibody conjugated to different fluorochromes. For example, there may be many different bottles of anti-CD3 available and some may look quite similar to staff that are new to the lab.

\section{DATA ANALYSIS}

The use of greater numbers of informative antibodies results in a geometric increase in the amount of information generated from the data. The ability to gain more information from a smaller amount of clinical material should reduce the number of inadequate results from studies performed on specimens containing low numbers of cells, such as cerebral spinal fluid, fine needle aspirates and paediatric samples. The use of increased numbers of antibodies in a single tube results in fewer tubes being processed, reduced sample volume, the time needed for data acquisition, and the time needed to process and acquire the specimen. Also, the use of more antibodies per tube means that redundancies (such as for CD45 used to identify leucocytes) within panels are reduced, leading to a more cost effective analysis. Fewer tubes and smaller samples allows for the routine acquisition of larger numbers of events, resulting in smaller coefficients of variation and more accurate data collection, particularly when analysing rare events. The disadvantages of multicolour analysis are: higher instrument costs; more specialised and costly reagents; the greater expertise needed to understand the pitfalls in analysis; and more complex data analysis. Software tools to assist data analysis are being developed, including the possibility of overlaying data from several individual tubes and to create libraries of normal and neoplastic antigen expression patterns. ${ }^{11}$

Nonetheless, at the moment, correct data analysis relies on the experience of the analysts, their knowledge of the normal antigen expression patterns and the abnormalities that may be observed in a variety of haematological malignancies. ${ }^{12-14}$ The analyst still needs to be sufficiently experienced to recognise when an instrument is not functioning correctly, and the incorrect use of compensation for spectral overlap is a major cause of false results. Software and reagents are available to help with compensation and instrument set-up. ${ }^{75}$ Clearly, it takes time for staff to acquire the appropriate level of experience to deal with the problems associated with instrument set-up and to recognise all the different expression patterns found in abnormal haematological cells. For this reason, as stated previously, there is a major need for standardised national and international training programmes.

\section{INTERPRETATION AND REPORTING}

The reporting of results varies from laboratory to laboratory and at present there is no consensus, although limited recommendations have been published. ${ }^{16}$ The amount of information is increasing and small populations of cells are more readily detectable than they were a few years ago. In bone marrow, it is now common to find small populations of immature or neoplastic cells, but their clinical significance may not be known and there is often uncertainty regarding how to report such findings.

Flow cytometry results are often still reported as percentages of the mononuclear gate in some cases, or of all white blood cells in other cases. It is also common to report a cell of interest gate, followed by a description of the abnormal cell(s), their number within the total white blood cell or nucleated cell count, and a description of their light scatter properties and phenotype. Many more antigens are now used than a few years ago and their relevance needs to be clarified. Most laboratories are now providing a multidisciplinary Haematology Malignancy Diagnostic Service, in which a combined report is generated for bone marrow and tissue specimens based on (as relevant) immunophenotype, morphology, histology, molecular genetics and cytogenetics, with input from the clinician treating the patient. This is important as it ensures the correct interpretation of the data in its clinical context.

\section{QUALITY CONTROL}

The introduction of multicolour flow cytometry has brought with it the need for new quality control (OC) measures. With more than one laser in use, a daily $\mathrm{OC}$ protocol that encompasses system optimisation, sets laser delays and area scaling factors is needed. ${ }^{17}$ This requires a high level of cytometry expertise and is time consuming if done manually, although software-driven QC programmes that enable a swift daily set-up QC are available.

As described above, the use of antisera cocktails should minimise labelling errors. However, there are no published data on the stability of antibody cocktails, so any home-made cocktail would require validation prior to diagnostic use. It would also be prudent to record the lot numbers of all individual antibodies within any home-made cocktail, as well as the date or lot of the cocktail used for any given specimen. Finally, the lot to lot re-compensation of tandem antibodies needs a QC system that allows cross-tracing of lot usage and the compensation settings used at the time of analysis.

\section{SUMMARY}

The arrival of multicolour flow cytometry brings with it new technical issues that require appropriate attention and

\section{Take-home messages}

The reader needs to focus on issues relating to the use of tandem dyes, the need for more complex compensation protocols and the requirements for rare event analysis. 
awareness by users and data interpreters. Once suitable protocols are established, the introduction of multicolour flow cytometry can certainly benefit haematology-oncology diagnostics by providing a more comprehensive view of several populations and their maturation pathways in any given sample, detecting smaller subsets within larger populations, and increasing the amount of data derived from paucicellular samples and minimal residual disease analysis, thereby enabling improved data interpretation and increasing the amount of information gathered from diagnostic samples.

\section{Competing interests None.}

Provenance and peer review Commissioned; not externally peer reviewed.

\section{REFERENCES}

1. Greig B, Oldaker T, Warzynski M, et al. 2006 Bethesda International Consensus recommendations on the immunophenotypic analysis of hematolymphoid neoplasia by flow cytometry: recommendations for training and education to perform clinical flow cytometry. Cytometry B Clin Cytom 2007;72(Suppl 1):S23-33.

2. Lee JA, Spidlen J, Boyce K, et al. MIFlowCyt: the minimum information about a Flow Cytometry Experiment. Cytometry A 2008;73:926-30.

3. van de Loosdrecht AA, Alhan C, Béné MC, et al. Standardization of flow cytometry in myelodysplastic syndromes: report from the first European LeukemiaNet working conference on flow cytometry in myelodysplastic syndromes. Haematologica 2009:94:1124-34.

4. http://www.euroflow.org/ (accessed 22 Feb 2011)
5. Wood B. 9-color and 10-color flow cytometry in the clinical laboratory. Arch Pathol Lab Med 2006;130:680-90.

6. Roederer M. Spectral Compensation for flow cytometry: visualization artifacts, limitations, and caveats. Cytometry 2001:45:194-205.

7. Bayer J, Grunwald D, Lambert C, et al. Thematic workshop on fluorescence compensation settings in multicolour flow cytometry. Cytometry B Clin Cytom 2007; 72:8-13

8. Roederer $\mathbf{M}$, Kantor $\mathrm{AB}$, Parks DR, et al. Cy7PE and Cy7APC: bright new probes for immunofluorescence. Cytometry 1996:24:191-7.

9. Hulspas R, Dombkowski D, Preffer F, et al. Flow cytometry and the stability of phycoerythrin-tandem dye conjugates. Cytometry B 2009;75:966-72.

10. Le Roy C, Varin-Blank N, Ajchenbaum-Cymbalista F, et al. Flow cytometry APC-tandem dyes are degraded through a cell-dependent mechanism. Cytometry $A$ 2009:75:882-90.

11. Pedreira CE, Costa ES, Barrena S, et al. Generation of flow cytometry data files with a potentially infinite number of dimensions. Cytometry $A$ 2008;73:834-46.

12. Gorczyca W. Flow Cytometry in Neoplastic Haematology. London: Taylor \& Francis 2006.

13. Craig FE, Foon KA. Flow cytometric immunophenotyping for hematologic neoplasms Blood 2008:111:3941-67.

14. Swerdlow SH, Campo E, Harris NL, et al. WHO Classification of Tumours of Haematopoietic and Lymphoid Tissues. Lyon: International Agency for Research on Cancer, 2008.

15. Herzenberg LA, Tung J, Moore WA, et al. Interpreting flow cytometry data: a guide for the perplexed. Nat Immunol 2006;7:681-5.

16. Wood BL, Arroz M, Barnett D, et al. 2006 Bethesda International Consensus recommendations on the immunophenotypic analysis of hematolymphoid neoplasia by flow cytometry: optimal reagents and reporting for the flow cytometric diagnosis of hematopoietic neoplasia. Cytometry B Clin Cytom 2007;72(Suppl 1):S14-22.

17. Perfetto SP, Ambrozak D, Nguyen R, et al. Quality assurance for polychromatic flow cytometry. Nat Protoc 2006;1:1522-30.

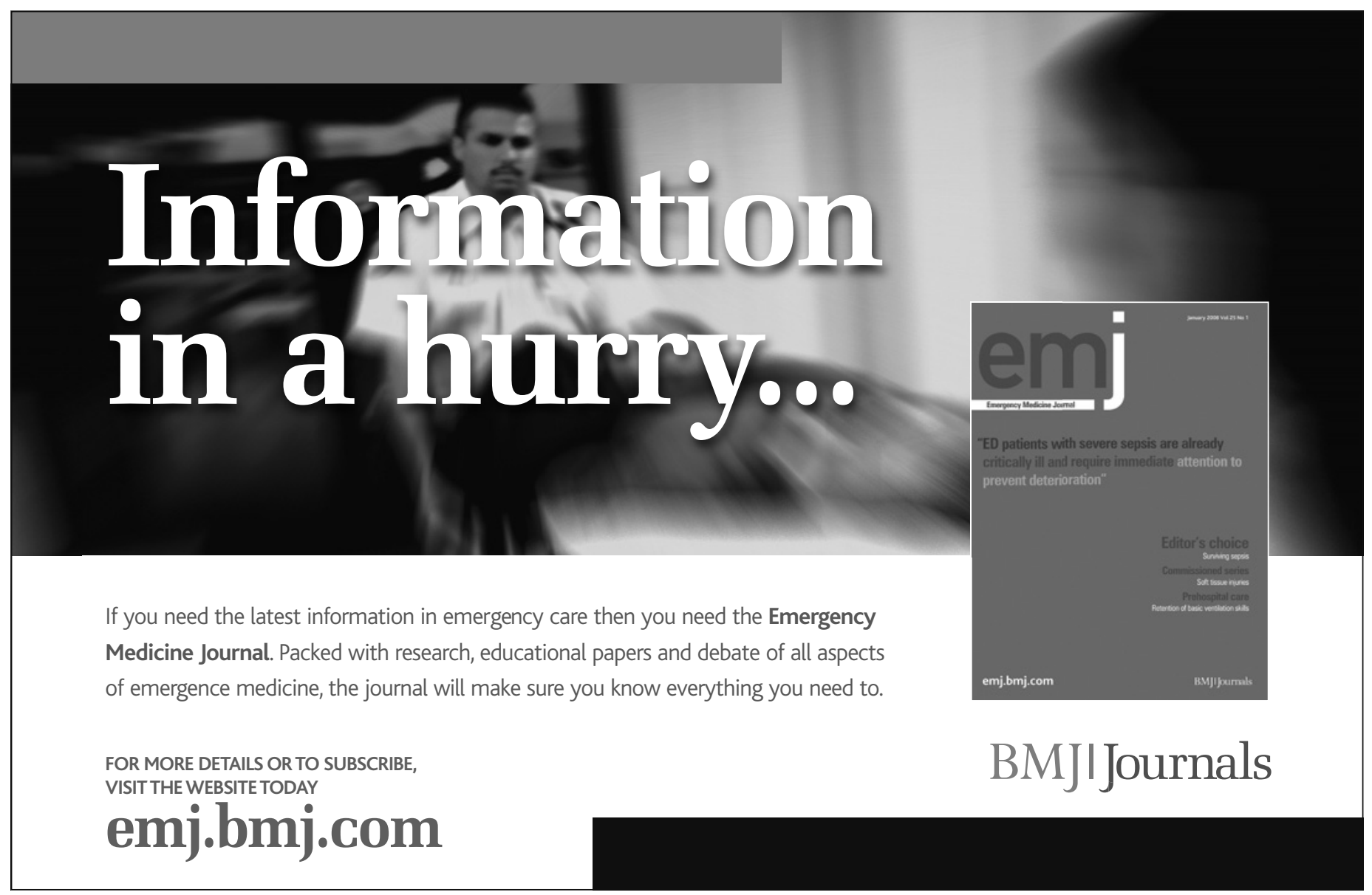

FOR MORE DETAILS OR TO SUBSCRIBE, VISIT THE WEBSITE TODAY 